\title{
Immune Genetic Algorithm for Optimizing Reinforced-Concrete Frame- Shear Wall Structure
}

\author{
Zheng Yinrui* and Zhu Jiejiang
}

Department of Civil Engineering, Shanghai University, Shanghai, 200072, P.R. China

\begin{abstract}
An immune genetic algorithm (IGA) is proposed to optimize the reinforced concrete (RC) frame-shear wall structures. Compared with the simple genetic algorithm (SGA), this algorithm has adaptive search capabilities for the future knowledge being used in the process of population evolution. Since the concrete grade of floors and the layout of walls are translated to binary codes, the implementation of this algorithm is not affected by the complexity of the structures. With I-typed vaccine, the continuous vertical stiffness of structure is ensured; With II-typed vaccine, the structures conforms to all the specifications which including floor shift angle, floor displacement ratio and period ratio. At the element level, the optimizing results satisfy all the specifications required by the current Chinese Codes. In this way, a computer program is created to get optimum design schemes.
\end{abstract}

Keywords: Immune genetic algorithm, optimization design, vaccines, RC frame-shear wall structure.

\section{INTRODUCTION}

Researches on the optimization for reinforcement concrete $(\mathrm{RC})$ frame-shear wall structures have been carried out by a lot of domestic and foreign scholars due to its wide application. The integration of shear walls and frames makes $\mathrm{RC}$ frame-shear wall structures have wide space and better earthquake-resistant abilities.

D. Z. Lin [1] insisted that the best design of RC frameshear wall structures should have the minimum earthquake action and meet the horizontal displacement constraints. S. J. Lin [2] and Z. F. Liu [3] stated that shear distribution of frames and shear walls are related to the stiffness characteristic value $\lambda$. They gave the formulas to describe the relationship between shear distribution and $\lambda$. They gave the formulas to describe the relationship between shear distribution and $\lambda$. Then they suggested that frames should be the second line of defense. F. Xie and P. S. Shen [4] concluded with their experiments that the axial pressure ratio of columns should be controlled to bring better dissipation capacity to the structures. K. J. Wang [5] put forward that the shear stiffness of beams is an important factor which affects the vertical stiffness of structures. All the above views provided qualitative ideas, but they lacked search strategy to realize automatic optimization. Design staffs have to adjust structures repeatedly in accordance with these views.

Recently, many researchers applied various bionic algorithms to optimize structures, e.g. I. Aydogdu and M.P. Saka [6] optimized a steel frame with ant colony algorithm, C. M. Chan [7] optimized the stiffness of a frame-shear wall structure via genetic algorithm, Q. Y. Xu [8] and L. N. Hou [9] optimized the large span column brace and ecological composite walls with the improved genetic algorithms.

Bionic algorithms, genetic algorithm as the representative, have parallel computing capacity. They can obtain the optimization space automatically and adjust the search direction adaptively. The initial points of the bionic algorithm are demanded to distribute evenly in the whole solution space, and search from all initial points at the same time, so they can search globally. In general, the bionic algorithm can get the global optimal solution of the problems whose solution set is non monotonic variation in the space, as shown in Fig. (1).

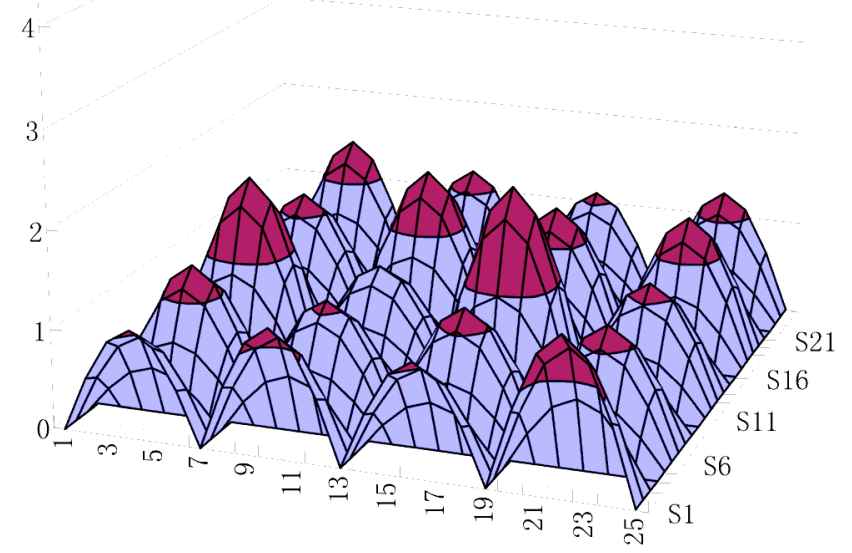

Fig. (1). Multi peak camber.

However, the traditional optimization method, such as optimality criteria method and mathematical programming method, only has one initial point. The search range is around the initial points or the optimal point in the previous round. It is more easily to converge to local optimal solution for its single and narrow search range. The effect of the wall 
layout on lateral resistant ability and the torsion resistant ability of the frame-shear wall structures is nonlinear and non monotonic, which is too complex to the traditional optimization methods, so the bionics algorithm should be used.

In December 1996, the concept Artificial immune system was firstly reported in an international symposium on immune system held in Japan. Artificial immune is inspired by the biological science. Biological Immunity means that body has protective physiological reaction. When the body is in contact with antigenic foreign matters such as various microorganisms, specificity will be produced to exclude them. The immune genetic algorithm (IGA) adds immune operator on the basis of simple genetic algorithm (SGA). The first step is to transform actual problems into appropriate codes and form a certain scale of population. Next, if some codes in an individual correspond to the actual structure that is incongruous with the known theory and experience during the evolutionary process, replace them with suitable codes. The basic process of IGA is shown in Fig. (2). Immune operator includes two processes Vaccination and Immune selection.

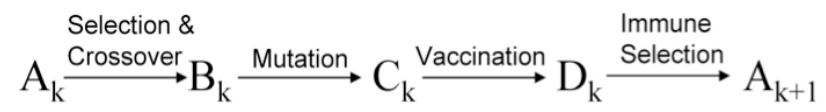

Fig. (2). Process of immune genetic algorithm.

The new codes applied to substitute for the original codes are called Vaccine. Vaccines for a particular antigen may be more than one. The one used to inject can be chosen at random or combine with several vaccines according to a logical order. The codes need to be replaced are called Antigen.

Immune selection is a process of judgment. If the fitness of an immunized individual is larger than the original one, the original one will be replaced by the immunized one, otherwise the original individual will be replaced by the corresponding one of the previous generation. The phenomenon that the fitness of son is smaller than his parent indicates that degeneracy is happened in crossover and mutation. Immune selection can avoid degeneracy.

Some studies on travelling salesman problem (TSP) [10] prove that IGA can improve the diversity and evolution trend of population. In 2013, H. S. Tang [11] and F. Li [12] successfully optimized trusses by immune clonal selection algorithm.

In this study, IGA is used to optimize the position and length of walls and concrete grades. Other variables include section sizes of columns, beams and shear walls. The objective is to reduce the cost of structures while meet all the constraints. The program based on IGA has shown good optimization effects and stable convergence.

\section{OPTIMIZATION MODEL}

\subsection{Objective Function}

The objective function is the cost of all beams, columns and shear walls, shown as Equation (1).

$\mathrm{W}=\sum_{\mathrm{i}=1}^{\mathrm{ne}} \mathrm{W}_{\mathrm{i}}+\sum_{j=1}^{n w} W_{j}$
Where, in the left of the plus is the total cost of all beams and columns, while in the right of the plus is the total cost of walls. $n_{e}$ is the total number of columns and beams and $n_{w}$ is the total number of walls. The objective function includes the cost of concrete, templates and steel bars with anchoring part. The cost of steel bars is calculated according to their number and diameter.

\subsection{Variables}

There are two types of variables. One is for optimization of walls layout, more specifically, it refers to the position and length of walls and concrete grade of each floor, shown in Fig. (3).

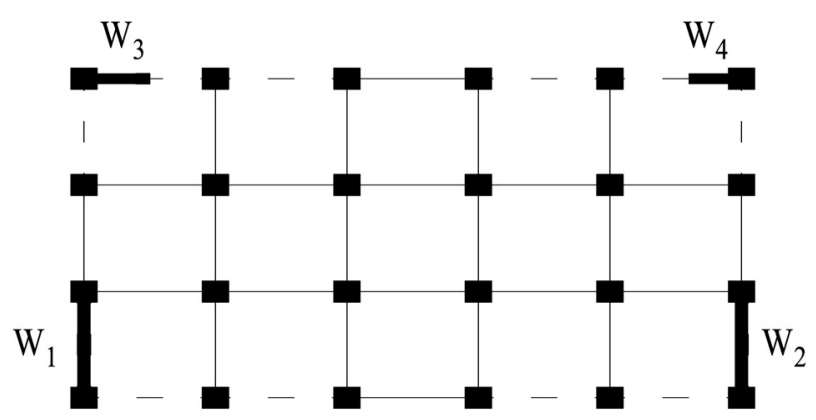

Fig. (3). Component of code.

Where, the grids expressed by the dotted line means shear wall can be layout here, while the grids expressed by solid lines means only beam can be placed here to satisfy the requirements of the architectural design. It does not mean all the grids with dotted line have to layout a wall. Which grids should be placed shear walls will be changed in the process of optimization. Once it is determined which grids placing shear walls, the others will be filled with beams. The shear walls can be arranged in the whole line, as $W_{1}$ and $W_{2}$ shown in Fig. (3), and can also be arranged in a part of the line, then the rest will be placed a beam, as $W_{3}$ and $W_{4}$ shown in Fig. (3).

The other is for optimization of section sizes, specifically standing for the height and width of beams sections, the length of columns sections and the thickness of walls. The beams and columns are all in the shape of rectangles.

\subsection{Constraints}

Constraints are also divided into two types for corresponding to the variables. One type consists of five constraints:

(1) The period ratio (the ratio of first torsion period and the first translation period) is less than 0.85 .

(2) The floor displacement angle (the ratio of floor displacement and floor height) less than 1/800.

(3) The floor displacement ratio (the ratio of maximum floor displacement and average floor displacement) of the given horizontal force [13] is less than 1.2.

(4) The concrete grade of a floor is not higher than the below floors. 
(5) The walls along the vertical direction are continuous.

The first three constraints called structure whole constraints $(S W C)$.

The other type is completely controlled by the section sizes and made up of three constraints:

(1) All members should have enough bearing capacity.

(2) All members should satisfy all the detailing requirements, such as the reinforcement ratios, the axial compression ratio, the reinforcement diameters and reinforcement spacing. These detailing requirements are all from Chinese codes GB50011-2010, GB50010-2010 and JGJ3-2010.

(3) The section sizes of columns and walls should satisfy equations (2-4).

$b_{\mathrm{x}, \mathrm{i}} \geq b_{\mathrm{x}, \mathrm{i}+1}$

$b_{y, \mathrm{i}} \geq b_{y, \mathrm{i}+1}$

$t_{\mathrm{i}} \geq t_{\mathrm{i}+1}$

In equations (2-4), every symbol is expressed as follows:

$b_{x, \mathrm{i}}, b_{x, i+1}, b_{y, \mathrm{i}}, b_{y, i+1}$--- the section length of columns at same position in floor $i$ and floor $i+1$;

$t_{\mathrm{i}}, t_{i+1}{ }^{---}$the thickness of walls at same position in floor $i$ and floor $i+1$.

\section{IGA FOR RC FRAME-SHEAR WALL STRUC- TURES}

\subsection{Selection Operator}

Roulette wheel selection is used as the selection operator. The basic idea of roulette wheel selection is that the selection rate is proportional to the fitness of an individual. The sum of the selection rate for all individuals is $100 \%$.

The specific operation is as follows: Determine the selected rate $p_{\mathrm{s}}$ of each individual according to the fitness in the previous round, shown as Equation. (5).

$$
p_{s, i}=\frac{F_{i}(A)}{\sum_{k=1}^{n} F_{\mathrm{k}}(A)}
$$

Where,

$$
F_{i}(A) \text { — } \text { the fitness of individual } i \text {. }
$$

Then generate a random number $\alpha(\alpha \in[0,1])$. The selected individual $k$ satisfies Equation. (6-7).

$$
\begin{gathered}
\rho_{k-1}<\alpha<\rho_{k} \\
\rho_{k}=\sum_{i=1}^{k} p_{s, i}
\end{gathered}
$$

Each selection operation will produce a new random number. The selection operator will not stop working until the number of individuals being selected is equal to the pop- ulation. Obviously, the individuals with high fitness may be selected repeatedly, and those with low fitness may be completely abandoned, which is just like the survival of the fittest.

\subsection{Crossover Operator}

The single point crossover operation is adopted. The specific operation is as follows: Selected two parent individuals by selection operator from previous generation. Then generate a crossover point randomly. The coding behind the crossover point will be exchanged. Then two son individuals are formed and added in the current generation, shown as follows.

Two parent individuals are:

Parent 1, 0111010011;

Parent 2, 1000101100.

The crossover point is after the seventh bit. Then Two son individuals are:

Son 1, 0111010100 ;

Son 2, 1000101011.

In order to maintain the stability of the population, the crossover rate $p_{\mathrm{c}}$ is set before the evolution. $p_{\mathrm{c}}$ can avoid the individuals with high fitness being destroyed by the following ways: A random number $\beta(\beta \in[0,1])$ is generated before the crossover operator works. If $\beta \leq p_{c}$, the crossover operator will be called. Otherwise, parent individuals will add in the current generation as son individuals.

\subsection{Mutation Operator}

The work method of the mutation operator is to scan all bits of the coding and decide which bits will be mutated. When checking a coding, mutation operator generates a random number $\gamma(\gamma \in[0,1])$. If $\gamma<p_{m}\left(p_{m}\right.$ is the mutation rate which is preset), the bit will be mutated, which specifically means the bit will set to 1 if it is 0 , or will set to 0 if it is 1 . Mutation operator is highly destructive, because it may change several bits in a coding, so the value of $p_{m}$ should be low.

\subsection{Genetic code component}

The introduced IGA used binary codes, roulette wheel selection and single point crossover conforms to the schema theorem [14] of SGA, shown as equations (8-9). The schema theorem indicates the schema has low schema order [14], short schema defining length [14] and whose fitness larger than the averaged fitness will be increased exponentially in the offspring.

$$
\begin{aligned}
& \mathrm{m}(\mathrm{H}, \mathrm{t}+1) \geq \mathrm{m}(\mathrm{H}, 0) p_{e}^{t} \\
& p_{e}=(1+\mathrm{c})\left[1-p_{c} \frac{\delta(H)}{l-1}-O(H) p_{m}\right]
\end{aligned}
$$

In Equation (5), every symbol is expressed as follows: $m(H, 0) 、 m(H, t+1)---t h e$ number of individual has schema $H[14]$ in the initial generation and generation $t+1$; 
$p_{e^{---i s}}$ the survival rate of schema $H$.

In Equation (6), every symbol is expressed as follows:

c ---the different value between the averaged fitness of individuals with schema $H$ and the average fitness of all individuals (not considering the differences between each generation);

$\delta(H)^{---}$-the schema defining length of schema $H$;

$l$---the length of an individual;

$O(H)^{---}$-the schema order of schema $H$;

$p_{m}{ }^{---t h e ~ m u t a t i o n ~ r a t e . ~}$

The genetic codes contain four types of information in which horizontal layout of walls has the most obvious impact on $S W C$, followed by the length of walls, the vertical layout of walls and concrete grades. The component of codes should keep the horizontal layout of walls diversified during evolutionary process to obtain the best solution in the whole solution range, which means $p_{s}$ of the schema of the walls horizontal layout should be low. The code component is shown in Fig. (4).

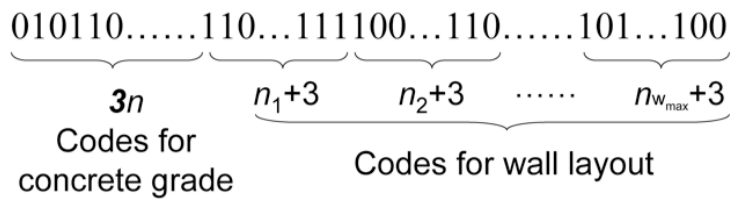

Fig. (4). Component of code.

Where, $W_{\max }{ }^{---}$the total number of grids that can set walls in the first floor.

The concrete grade of a floor is represented by 3 bits. From the ground floor to the top floor, $3 n$ ( $n$ is the total number of floors) bits are needed. That is at the front part of the codes. The range from 000 to 111 is translated to from $\mathrm{C} 30$ to $\mathrm{C} 65^{[15]}$. The followed codes represent the walls layout of a grid which is expressed by $n_{i}+3$ bits ( $n_{i}$ refers to the total number of floors in the grid). The front $n_{i}$ bits indicate which floors of a grid have walls. For example, 1100 means first and second floors have walls but third and fourth floors have no walls. The last 3 bits represent the length of a wall. 000 refers to the minimum length $L_{\min }, 111$ refers to the maximum length $L_{\max }$, and others should be calculated by linear interpolation. For example, if $L_{\min }$ is $800 \mathrm{~mm}$ and $L_{\max }$ is $2000 \mathrm{~mm}$, then 010 can be calculated as Equation. (10).

$L=L_{\min }+2\left(L_{\max }-L_{\min }\right) / 7=1171.43 \mathrm{~mm}$

$L$ has to be a multiple of $50 \mathrm{~mm}$, so the wall length represented by 010 is $1150 \mathrm{~mm}$.

The maximum of $O\left(H_{h}\right)$ is $W_{\max }$, where $H_{h}$ is the schema of the walls horizon layout. The maximum of $O\left(H_{v}\right)$ is the total number of floors in a grid, where $H_{v}$ is the schema of the walls vertical layout in a grid. The maxi- mum of $O\left(H_{l}\right)$ is 3, where $H_{l}$ is the schema of wall length. The maximum of $O\left(H_{c}\right)$ is 3 , where $H_{c}$ is the schema of concrete grade of a floor.

In conclusion, schema order is related to the building scheme, which cannot be changed in the genetic codes. However the schema defining length can be adjusted. The schema defining length of every schema is shown as Equations. (11-14).

$\delta\left(H_{h}\right)=\sum_{i=1}^{W_{\text {max }}}\left(n_{i}+3\right)$

$\delta\left(H_{\mathrm{v}}\right)=n_{i}$

$\delta\left(H_{1}\right)=3$

$\delta\left(H_{\mathrm{c}}\right)=3 n$

Obviously, $\delta\left(H_{h}\right)$ is larger than $\delta\left(H_{v}\right), \delta\left(H_{l}\right)$ and $\delta\left(H_{c}\right)$. The component of genetic codes, as shown in Fig. (2), makes $H_{h}$ has longer defining length, while $H_{v}, H_{l}$, $H_{c}$ have shorter defining length, which keep the mainly factor diversified. Thus the search is less likely to converge to the local optimal solution.

\subsection{The Fitness Function}

The fitness function is shown as Equations. (15)-(16).

$\mathrm{F}(A)=\mathrm{C} / f(A)$

$f(A)= \begin{cases}\mathrm{W} & \text { When meet SWC } \\ 2 \mathrm{~W} & \text { When not meet SWC }\end{cases}$

Where,

W ---shown as Equations. (1);

$C$---a large preset number. Specifically, $C$ is 3 times of the maximal $f(A)$ of the initial generation.

When an individual does not meet $S W C, f(A)$ is equal to $2 \mathrm{~W}$ to reduce the selection rate.

\subsection{Immune Operator}

Immune operator has two functions. One is to avoid some invalid operation. The other is to improve the population fitness. There are three vaccines during the evolutionary process.

(1) Vaccine for the vertical layout of walls.

The vaccine for the vertical layout of walls is to eliminate the situation that the walls is not continuous along the vertical direction, shown as the Fig. (5(a)-5(b)), because it is invalid to check thus individuals. The codes 1010 and 0011 which represent the situation 5(a) and 5(b) are antigens. There are several corresponding vaccines. The codes 1100 and 1110 which represent the situation shown in Fig. (5(c)) 5(b)) are two vaccines can be chosen. 


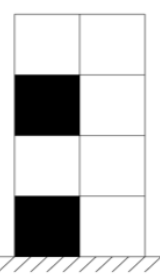

(a)

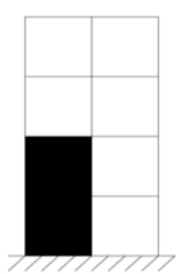

(c)

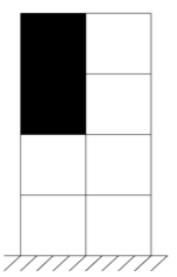

(b)

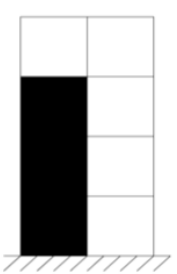

(d)
Fig. (5). The antigen and vaccine of wall layout.

In the introduced IGA, a vaccine will be chosen at random to inject. In other words, the antigen codes will be replaced by the vaccine codes.

(2) Vaccine for concrete grades.

The vaccine for concrete grades is to eliminate the situation that concrete grade of a floor is higher than the below one, as checking such individual is also invalid. The vaccine codes for the concrete grade of a floor are the codes for the concrete grade of the adjacent below floor. For example, if codes for concrete codes appear 100110, the antigen codes are 110 and the vaccine codes will be 100 .

(3) Vaccine for $S W C$.

The vaccine for $S W C$ is to make individuals meet $S W C$. The vaccine is to improve the fitness of the population, since $f(A)$ is equal to $2 \mathrm{~W}$ when an individual does not meet $S W C$.

In this part, the $S W C$ vaccine library is introduced. The codes for walls layout (as shown in Fig. 2) of an individual that meeting SWC will be added to the library. When the library reaches a certain scale, such as the total number of vaccine is more than 50 percent of the population, vaccination begins. Whether an individual which not meeting $S W C$ will be injected or not depends on the vaccination rate $p_{v}$. The suitable value of $p_{v}$ is between 0.2 to 0.3 , as the premature phenomenon may appear if $p_{v}$ is high. The vaccine selection is also used roulette wheel selection. The Vaccine for $S W C$ essentially makes the individual that meeting $S W C$ have one more chance to be checked after changing concrete grades. In a certain extent, it keeps the population diversity and improves the population fitness because the influence of the concrete grades on the $S W C$ is very small.

The vaccine for vertical layout of walls and concrete grades can be formed before checking the individual, so they are called I-typed Vaccine. The vaccine for SWC must be formed after checking the individual, so it is called II-typed Vaccine. The introduced IGA does not need Immune Selection, as I-typed Vaccine is to avoid invalid operations and IItyped Vaccine can improve the fitness of an individual in most cases. The process of the introduced IGA is shown in Fig. (6).

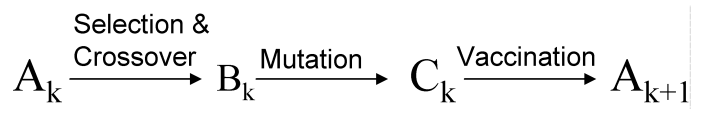

Fig. (6). The antigen and vaccine of wall layout.

\section{REALIZATION}

The introduced IGA is realized by creating a program by $\mathrm{VC}++$. The program creates an initial generation automatically according to the input population scale. Each individual in the initial generation is unique and will be injected I-typed Vaccine if necessary.

Optimization of layout is completed by IGA and optimization of section sizes is completed by the grid search algorithm [16]. The variables of section sizes are discrete and their steps are $50 \mathrm{~mm}$. Every rectangle section has 2 variables which the maximum and minimum values are preset. The solution range can be described as a grid space. Each point will be tested in the order shown in Fig. (7).

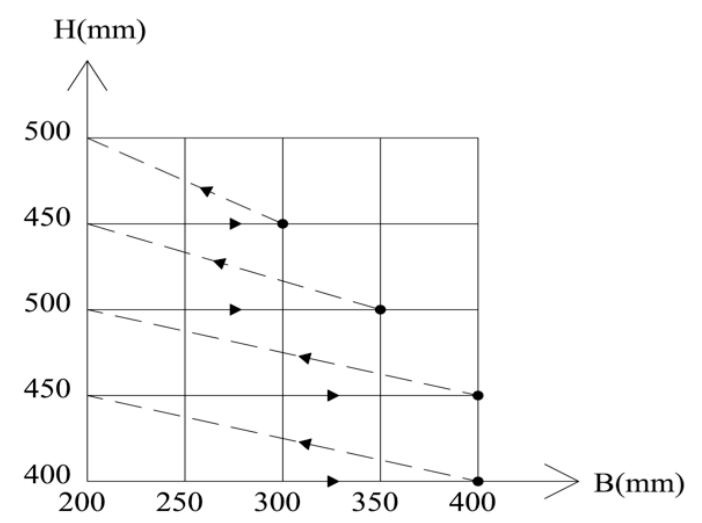

Fig. (7). The grid search method.

The point corresponding to the lowest cost is the optimal point. The optimization process of the thickness of walls is similar to that of section sizes of columns.

The evolutionary process of an individual is shown in Fig. (8). At last, the structure according to the individual with highest fitness is the optimal solution.

The optimization process of a structure which the wall layout has determined begins from the top floor, then work down floor by floor. The Equations. (2-4) are transformed as Equations. (17-19) in order to realize the section size is not larger than the one in the same position at the below floor.

$b_{\mathrm{x}, \mathrm{i}, \min }=b_{\mathrm{x}, \mathrm{i}+1, \mathrm{opt}}$
$b_{\mathrm{y}, \mathrm{i}, \min }=b_{\mathrm{y}, \mathrm{i}+1, \mathrm{opt}}$
$t_{\mathrm{i}, \text { min }}=t_{\mathrm{i}+1, \mathrm{opt}}$
Where

Where,

$b_{\mathrm{x}, \mathrm{i}, \mathrm{min}}, b_{\mathrm{y}, \mathrm{i}, \mathrm{min}}---$ the minimum section sizes of columns in floor $i$;

$b_{\mathrm{x}, \mathrm{i}+1, \mathrm{opt}}, b_{\mathrm{y}, \mathrm{i}+1, \mathrm{opt}}---$ the optimal section sizes of columns in floor $i+1$; 
Table 1. Unit price of material.

\begin{tabular}{|c|c|c|c|c|}
\hline \multirow{5}{*}{$\begin{array}{c}\text { Concrete } \\
\left(\mathrm{CNY} / \mathrm{mm}^{3}\right)\end{array}$} & C30 & 550 & 605 & 525 \\
\hline & C40 & 590 & 645 & 565 \\
\hline & C55 & 670 & 725 & 645 \\
\hline & C60 & 705 & 760 & 680 \\
\hline & C65 & 740 & 795 & 715 \\
\hline
\end{tabular}

$t_{\mathrm{i}, \min }-$--the minimum thickness of walls in floor $i$;

$t_{i+1, \text { opt }}{ }^{---}$the optimal thickness of walls in floor $i+1$.

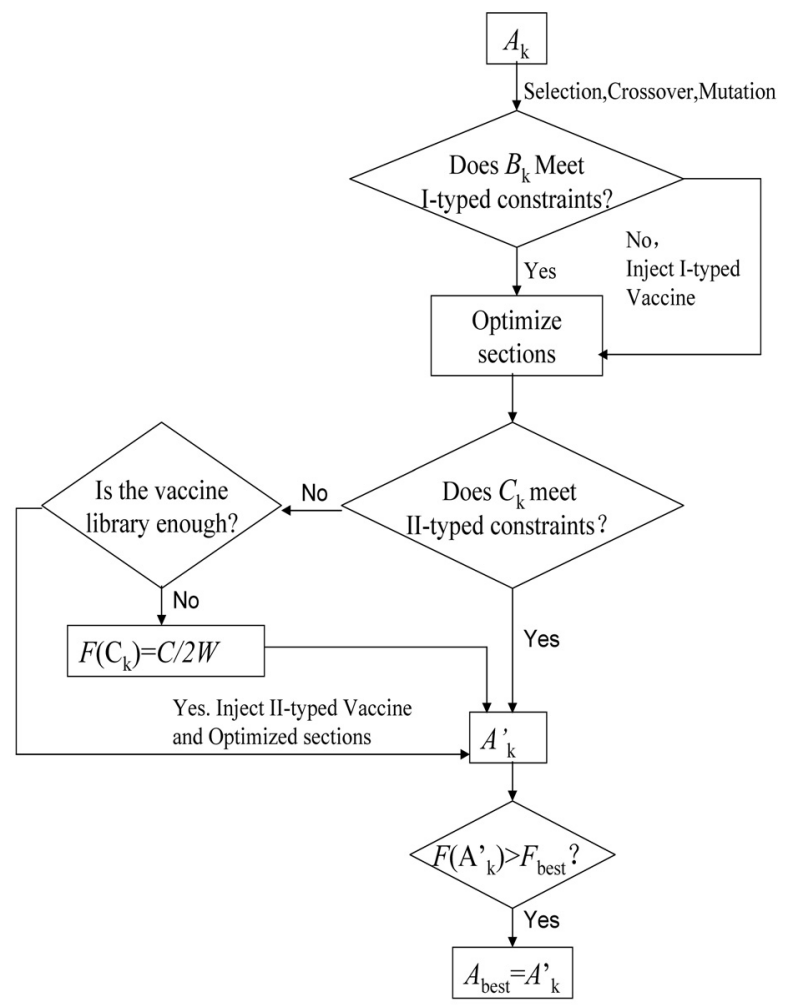

Fig. (8). Individual evolution.

\section{EXAMPLE}

There is a structure with 7 floors. The original plan of the structure is shown in Fig. (9). The floors are all $3000 \mathrm{~mm}$ high. The dead load on floor slabs is $8.0 \mathrm{kN} / \mathrm{mm}^{2}$ and the live load on floor slabs is $2.0 \mathrm{kN} / \mathrm{mm}^{2}$. The structure is located at Shanghai and the wind load and earthquake response is cal- culated by Chinese codes GB50009-2010 and GB500112010.

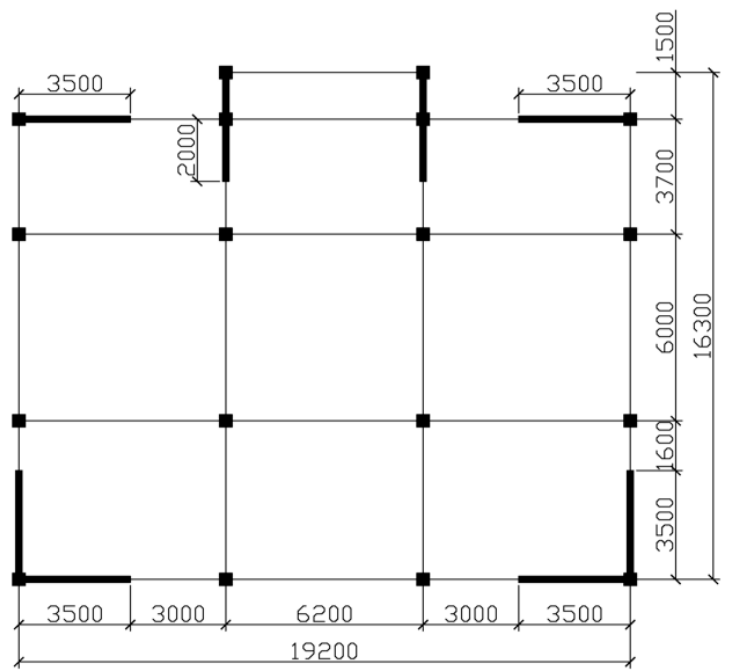

Fig. (9). Original structure plan.

The unit prices of material shown as Table $\mathbf{1}$.

There are 50 individuals in the population. The layout of walls in every floor keeps the same required by the architect.

After evolving 20 generations, The statistical data of the population is shown in Fig. (10). The beginning part of the average cost curve is high because the $f(A)$ of individuals not meeting $S W C$ have to be equal to $2 W$ as the $S W C$ vaccine library has not reach the enough scale. The structure corresponding to the lowest cost is shown in Fig. (11), which is the optimized structure. $S W C$ of the optimized structure are as follows.

(1) Period ratio is 0.56 .

(2) The maximum floor drift angle is $1 / 1177$ of the $\mathrm{X}$ direction earthquake response and 1/1393 of the Y-direction earthquake response. 
(3) The maximum floor displacement ratio is 1.01 of the $\mathrm{X}$-direction given horizontal force and 1.00 of the Ydirection given horizontal force.

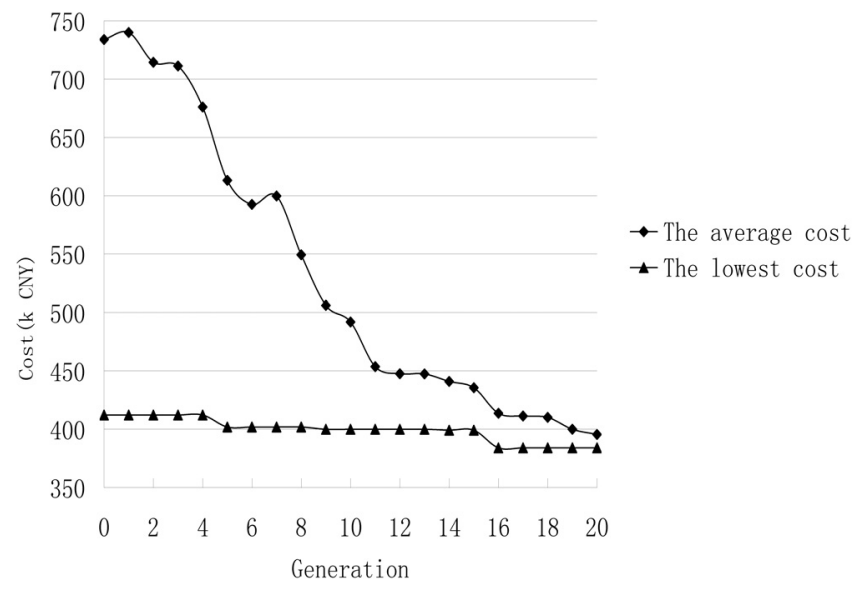

Fig. (10). Cost trend.

After optimizing, the concrete grades of all floors are C30. In the first and second floors, the section sizes of column 1 and 2 are $550 \mathrm{~mm} \times 550 \mathrm{~mm}$ and column 3 and 4 are $500 \mathrm{~mm} \times 500 \mathrm{~mm}$. In the third floor, the section sizes of column 1 and 2 are $500 \mathrm{~mm} \times 500 \mathrm{~mm}$ and column 3 and 4 are $450 \mathrm{~mm} \times 450 \mathrm{~mm}$. In the fourth floor, the section sizes of column 1, 2, 3 and 4 are $450 \mathrm{~mm} \times 450 \mathrm{~mm}$. The section sizes of other columns are all $400 \mathrm{~mm} \times 400 \mathrm{~mm}$. The columns numbers are shown in Fig. (11). The section sizes of the beams are all $250 \mathrm{~mm} \times 400 \mathrm{~mm}$. The thickness of walls are all $200 \mathrm{~mm}$.

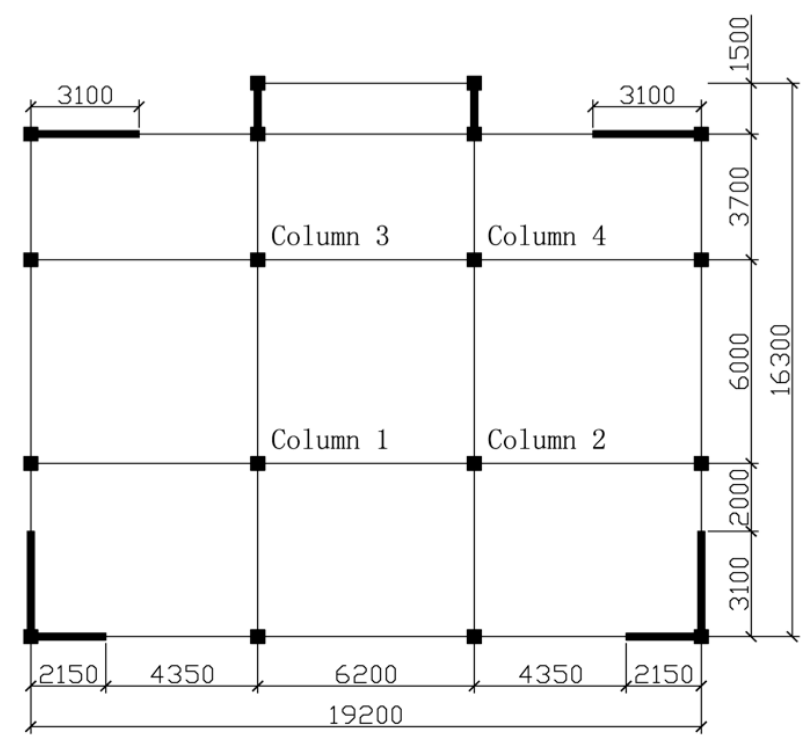

Fig. (11). Optimized structure plan.

The cost of original structure is $¥ 502,800$ and the cost of optimized structure is $¥ 383,900$. The cost has $23.6 \%$ reduction.

\section{CONCLUSION}

The most critical part of designing frame-shear wall structures is to design the layout of walls. The general approach is to adjust structures repeatedly, which requires a lot of manpower and time. In this study, a computer program is created to optimizing RC frame-shear wall structures via the introduced IGA realized designing the layout of walls automatically. IGA can improve the design efficiency and resolve two hard issues.

(1) The optimized structures meet $S W C$. In the evolutionary process, $S W C$ vaccine library is formed simultaneously without increasing computation, which realizing self learning.

(2) IGA can search in the whole solution range to avoid the scheme being just the local optimal solution due to the lack of experience and time of the designers. As proved by the example, the introduced IGA has good convergence. The structures optimized by the IGA are ideal solutions for their low costs.

\section{CONFLICT OF INTEREST}

The authors confirm that this article content has no conflict of interest.

\section{ACKNOWLEDGEMENTS}

This work was a grand special project, financially supported by Shanghai science and technology commission (No. D.10-0113-05-006).

\section{REFERENCES}

[1] D. Z. Lin. "A Method of Stiffness Optimization of the Frame-Shear Wall Structure", Journal of Heber institute of architectural engineering, vol. 18, no. 9, pp. 17-19, 2000.(In Chinese)

[2] S. J. Lin. "The Distribution of Seismic Shearing Force in FrameShear Wall Structure", Guangzhou Architecture. vol. 40, no. 4, pp. 2-9,2012.(In Chinese)

[3] Z. F. Liu, Z. L. Su, W. J. Yang. "Simplified method to distinguish the rational arrangement of shear walls in frame-shear wall structures". Building Structure, vol. 40, no. 4, pp. 103-106, 2010.(In Chinese)

[4] F. Xie, P. S. Shen. "Experimental Study of the Storey Modes of Frame-shear Wall Hybrid Structure", Journal of Human University(Natural Sciences), vol. 37, no. 11, pp. 12-17,2010.(In Chinese)

[5] K. J. Wang. "Geometrical design of vertical components of highrise frame-shear wall structures". Building Structure, vol. 40, no. S, pp. 74-76,2010. (In Chinese)

[6] I. Aydogdu, M.P. Saka. "Ant colony optimization of irregular steel frames including elemental warping effect". Advances in Engineering Software,vol. 44, no. 1, pp.150-169,2012.

[7] C. M. Chan, K. M. Wong. "An Efficient Hybrid Genetic Algorithm for Topological Layout Optimization of High-rise Residential Buildings", in Proc. 4th China-Japan-Korea Joint symposium on Optimization of Structural and Mechanical Systems, Kunming, 2006, 397-402.

[8] Q. Y. Xu, A. Q. Li,etc. "Research on location optimization of energy dissipation braces between columns of long-span hangar based on improved genetic algorithm", China civil engineering journal, vol. 46, no. 6, pp. 35-43,2013.(In Chinese).

[9] L. N. Hou, W. Huang,etc. "Optimum design of ecological composite wall based on modified Genetic Algorithm", China civil engineering journal, vol. 43, no. S, pp. 416-420,2010.(In Chinese). 
[10] L. C. Jiao, H. F. Du, etc. Calculation, learning and recognition of Immune optimization, Beijing: Science Press, 2006, pp. 8-9. (In Chinese).

[11] H. S. Tang, C. Y. Hu, S. T. Xue. "Immune clonal selection algorithm for truss structure multi-objective optimization", Journal of Hunan University (Natural Sciences), vol. 40, no. 5, pp. 1823,2013.(In Chinese).

[12] F. Li, H. S. Tang,etc. "Immune clonal selection algorithm for truss structure optimal design", Journal of Tongii University (Natural science), vol. 38, no. 9, pp. 1261-1265,2010. (In Chinese).
[13] GB50011-2010, Code for seismic design of buildings (in Chinese), Bureau in Chinese National Standard, Beijing (2010).

[14] GB50011-2010, Code for seismic design of buildings[S].

[15] GB50010-2010, Code for design of concrete structures (in Chinese), Bureau in Chinese National Standard, Beijing (2010).

[16] J. J. Zhu, Y. R. Zheng. "The multilevel optimal design of space RC frame structures and its program development", Building Science, vol. 25, no. 1, pp. 91-95,2009. (In Chinese).

Received: May 26, 2015

Revised: July 14, 2015

Accepted: August 10, 2015

(c) Yinrui and Jiejiang; Licensee Bentham Open.

This is an open access article licensed under the terms of the (https://creativecommons.org/licenses/by/4.0/legalcode), which permits unrestricted, noncommercial use, distribution and reproduction in any medium, provided the work is properly cited. 